\title{
EndoConf: real-time video consultation during endoscopy; telemedicine in endoscopy at its best $\square$
}

다)(용

\author{
Authors \\ Meyer ${ }^{1,2}$ () \\ Institutions \\ 1 Department of Surgery, Odense University Hospital, \\ Denmark \\ 2 Department of Clinical Research, University of Southern \\ Denmark, Denmark \\ 3 Department of Social Medicine \& Public Health, \\ Pomeranian Medical University, Szczecin, Poland
}

Ulrik Deding ${ }^{1,2} \odot$, Anders Høgh ${ }^{1}$, Niels Buch ${ }^{1}$, Anastasios Koulaouzidis ${ }^{3} \odot$, Gunnar Baatrup ${ }^{1,2}$, Thomas Bjørsum-

submitted 12.4 .2021

accepted after revision 2.7 .2021

\section{Bibliography}

Endosc Int Open 2021; 09: E1847-E1851

DOI 10.1055/a-1548-1631

ISSN 2364-3722

(c) 2021. The Author(s).

This is an open access article published by Thieme under the terms of the Creative Commons Attribution-NonDerivative-NonCommercial License, permitting copying and reproduction so long as the original work is given appropriate credit. Contents may not be used for commercial purposes, or adapted, remixed, transformed or built upon. (https://creativecommons.org/licenses/by-nc-nd/4.0/)

Georg Thieme Verlag KG, Rüdigerstraße 14,

70469 Stuttgart, Germany

Corresponding author

Thomas Bjørsum-Meyer, Department of Surgery, Odense Universitetshopital, Winsløve 4, Odense 5000, Denmark Fax: +0065413035

thomas.bjoersum-Meyer@rsyd.dk

\section{ABSTRACT}

Background and study aims The aim of this study was to introduce EndoConf, a reliable and easy-to-use tool capable of optimizing clinical care in endoscopy by reducing the number of repeat endoscopy procedures, providing continuous on-the-job clinical education, and allowing a smooth transition to the next level of artificial intelligence-supported systems.

Patients and methods We prospectively developed and improved a real-time conference system (EndoConf). EndoConf enables endoscopists to contact on-demand and in real time experienced endoscopists across other sites. After the initial introduction period, we registered all EndoConfassisted procedures from our unit (Surgical Department of Odense University Hospital) over a 3-month period (Autumn of 2019).

Results Of 84 EndoConf-supported procedures, 58 were eligible for further analysis. Eventually, 38 calls were made, of which only four were technically of low quality (10.5\%) while three were not answered (7.9\%). Of the 35 (92.1\%) completed EndoConf calls; 24 were referred for endoscopic mucosal resection, six were referred for transanal microsurgery preceded by transrectal ultrasonography and three were referred for multidisciplinary conference, whereas in two cases, the lesion was resected during EndoConf.

Conclusions We found the EndoConf system to provide support that could reduce the number of unnecessary repeat endoscopic procedures while at the same time ensuring avoidance of any hazardous attempt at polypectomy.

\section{Introduction}

Millions of endoscopic procedures are performed around the world each year. It is important to provide high-quality endoscopy with optimal decision-making for precision in care delivery and to ease overburdened healthcare systems. Furthermore, with broader adoption of systematic colorectal cancer (CRC) screening [1], there is a continuous demand for recruiting screening colonoscopists while maintaining high standards of care for all other non-screening endoscopic procedures [2]. The latter include existing polypectomy variations, with multi- ple techniques available and a wide range of experience among endoscopists and training programs [3].

Furthermore, over the last decade, different polypectomy methods and techniques have been developed, and there has been an increase in the incidence of surgeries for non-malignant polyps [4]. Therefore, endoscopists need to be familiar with these techniques and comfortable deciding when to proceed and when to refer, which guarantees the selection of the best possible care for each patient [5]. Familiarity with and training in endoscopic techniques can also affect the outcome for patients [6]. The ever-increasing number of neoplastic le- 
sions managed by endoscopic techniques instead of open or laparoscopic surgery and the multiple and intricate endoscopic classifications raise the complexity of decision-making in colonoscopy. This led to the development of the Danish SATC center [7]. This paper aims to present the results of the SATC center's introduction of a reliable and easy-to-operate conference tool to support optimal clinical care, reduce the number of repeat endoscopy procedures, provide continuous on-the-job professional development, and ease the transition to the next level of artificial intelligence (AI)-supported systems.

\section{Patients and methods}

The EndoConf system was implemented in our surgical department, which is located in three separate units (Odense, Svendborg, and Nyborg), providing cover to the island of Funen, Denmark. It delivers 10,000 colonoscopies/year for diagnosis and treatment of colorectal neoplasia. In collaboration with Odense University Hospital's medicotechnical department, we prospectively developed and improved a real-time (RT) conference system (EndoConf). EndoConf enables our department's endoscopists to contact experienced endoscopists, working across other sites, in real time (RT) during endoscopy and to send high-resolution images/video feed and get a response in a short time.

This paper presents the results of a feasibility study performed when EndoConf was implemented for clinical use and assesses its application, quality of calls, and outcomes.

\section{Workflow}

During a colonoscopy, the EndoConf system enables the endoscopist to consult an expert endoscopist when faced with troublesome decision-making regarding diagnostics and handling of colorectal (CR) polyps. An experienced endoscopist staffed the on-call system during working hours (Monday-Friday until 15.30). The doctor on call was able to go about usual practice and change location as long as the tablet stayed with him. The audio-video-link carried an audio signal, a video signal from the endoscopy room, and a video signal from the endoscope ( $\mathbf{F i g .}$ 1).

When the endoscopist needed an expert opinion, one of the nurses could set up a RT video link through a mobile workstation ( $>$ Fig. 2) to the expert endoscopist on duty, who could livestream the endoscopy from a portable mobile device (tablet) ( Video 1).

Our department operates three endoscopic units at three different locations, 25 to $40 \mathrm{~km}$ apart. One unit performs advanced colonoscopies and procedures, such as endoscopic ultrasound, endoscopic mucosal resection (EMR), endoscopic submucosal dissection (ESD), endoscopic full-thickness resection, transanal endoscopic microsurgery (TEM), and hybrid procedures. This unit has surgical facilities that facilitate immediate rescue surgery in case of perforation, bleeding, or other adverse events and it facilitates high-risk endoscopic procedures. The same unit contains the regional SATC-C center for advanced adenomas and early colorectal cancers [5]. The center

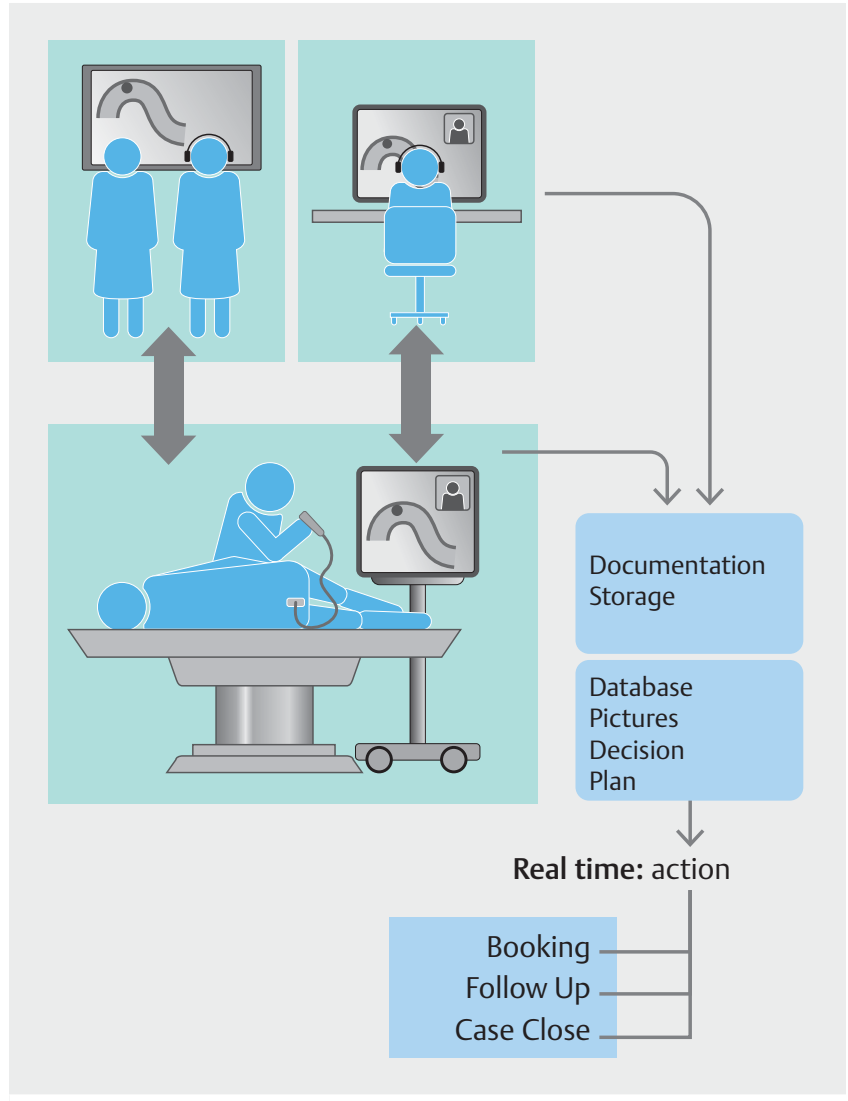

- Fig. 1 EndoConf system workflow.

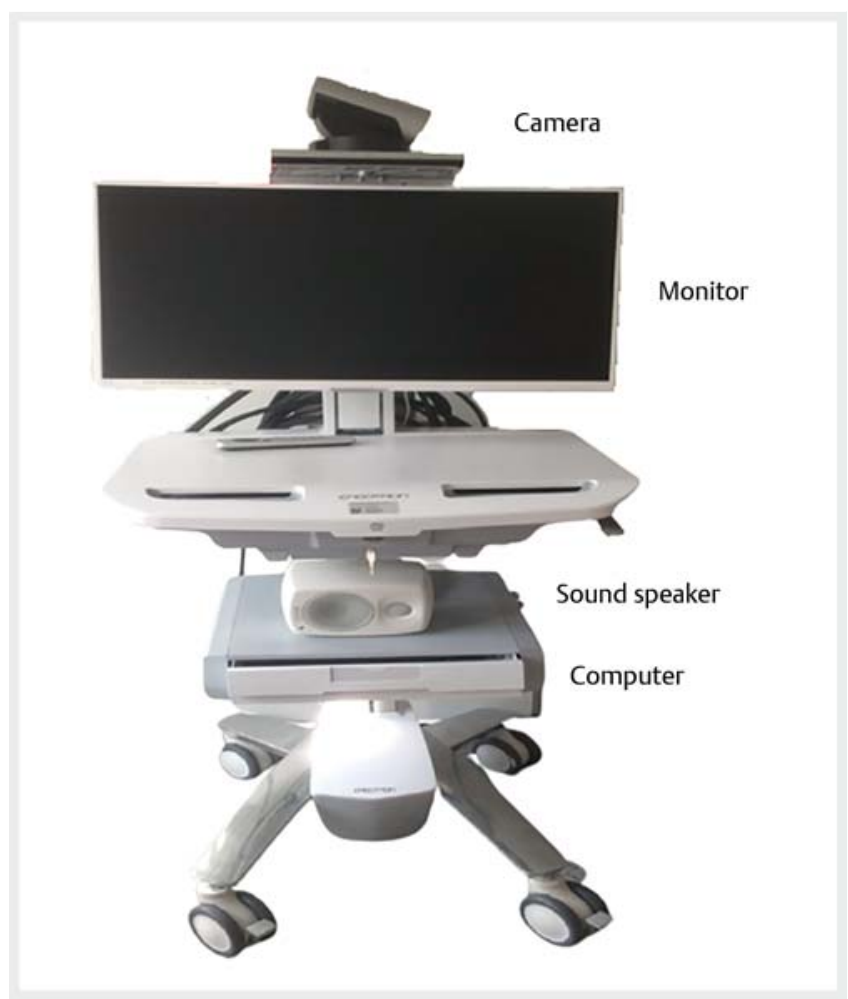

- Fig. 2 Mobile workstation (Endotrolley) at the endoscopy unit enabling real-time video link. 


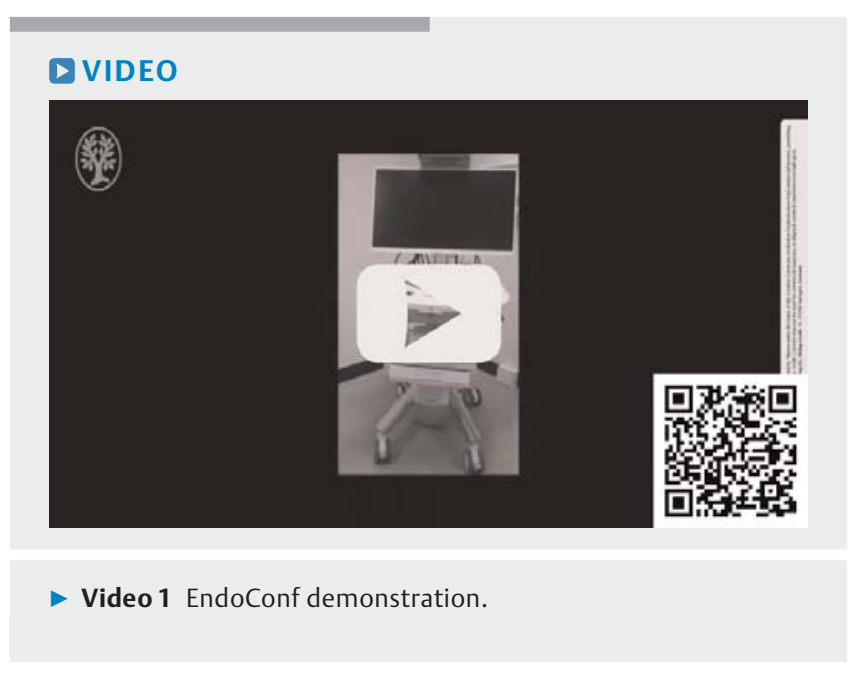

is dedicated to ensuring that state-of-the-art treatment of uniform quality if provided throughout the region of Southern Denmark, which includes other four independent University Hospitals at six different locations with colonoscopy activity. The SATC-C center conducts courses, hands-on training, symposia and fellowships for research and development. All colonoscopists in the region have to attend the introductory colonoscopy course provided.

EndoConf was initially implemented at one unit (Nyborg) in three to four endoscopy rooms daily (Monday-Friday). This was expanded with two to three endoscopy rooms at two other units (Svendborg and Nyborg). On average, 10 colonoscopies were performed daily in each endoscopy room. The endoscopists at the involved units were told about EndoConf before it was implemented.

EndoConf was funded by SATC-C, external funds and the Region of Southern Denmark. Setup and maintenance of the EndoConf was provided by the local medicotechnical department.

The long-term aims of introducing EndoConf were to:

1. Enable support of novice endoscopists if experienced endoscopists are not available on site.

2. Reduce polypectomy attempts during diagnostic colonoscopy in cases where polypectomy complexity has been underestimated.

3. Reduce the number of unnecessary referrals for a repeat colonoscopy and polypectomy if polypectomy technical requirements had been overestimated.

4. Promote in-house implementation of new techniques learned during SATC-C courses.

5. Assist in delivering courses by supporting transmission of live procedures to the teaching facilities.

After the initial introduction period, we registered all EndoConf-eligible procedures from the unit, i. e., colonoscopies ending in referral for EMR/ESD over 3 months in the fall of 2019. From all referrals, the authors reviewed whether the index colonoscopy was performed in an operating room with EndoConf available. In case EndoConf had not been used, the referring doctor received a reminder about availability of the system. The date of the procedure, EndoConf calls made, call quality, experience of the endoscopist, result/referral following colonoscopy, and location of any neoplasia were documented. This was mainly done to identify any systematic IT difficulties that needed to be addressed before implementing the system throughout the Southern Denmark region and enabled evaluation of its use.

\section{Ethical considerations}

Because EndoConf was implemented as a novel tool, not a research project, for education and improved decision-making in line with the usual bedside attendance by an experience endoscopist, informed consent from patients and approval from the local ethical committee were not required. Prior to each EndoConf procedure the patient, if not sedated, was informed about the need for a RT video conference to discuss the best treatment or proper referral.

\section{Results}

The first EndoConf call was conducted successfully on December 12, 2018, and the system was then introduced in our surgery department by experienced endoscopists from the SATCC [1]. During a 3-month period, we prospectively registered 84 EndoConf-eligible procedures. Of them, 64 were in locations where the EndoConf equipment was available. In six cases, the endoscopist was one of the expert endoscopists on call for the EndoConf system; therefore, there was no need to make a call. Eventually, 58 colonoscopies were supported by the EndoConf conference equipment. In this group of colonoscopies, 38 procedures (65.5\%) had EndoConf calls made; four were of low quality technically (10.5\%) and three were not answered (7.9\%) ( Fig. 3). No answer to calls occurred due to connection problems at the receiving end or if the expert on call was preoccupied by other conferences. Of the 35 completed EndoConf procedures, 24 were referred for EMR; six were referred for TEM preceded by transrectal ultrasonography; three were referred for multidisciplinary conference; while in two cases, it was possible to resect the lesion under the guidance of the specialist who answered the call. The neoplasia location was right-sided in 12 cases, left-sided in 11, and unknown in 10 (two individuals had multiple neoplasias). Senior registrars or consultants in gastrointestinal surgery had requested $74 \%$ of the EndoConf calls. No unintended consequences were registered.

\section{Discussion}

We found EndoConf to be a helpful tool for supervising and educating our endoscopists. With modifications to broadband speed and dedicated broadband prioritized in the Region's Network, EndoConf became a well-functioning tool, with short dial time and high-quality sound and images. EndoConf enables discussions about lesion characterization and management in RT instead of the common practice of storing still pictures for subsequent discussion and evaluation by senior endoscopists.

In the course of putting EndoConf into practice, ethical considerations are mandatory. During a live call with discussions of 
84 EndoConf eligible procedures at surgical unit

20 EndoConf equipment not available

64 with the option to conduct a conference call

6 Expert endoscopist was conducting the

colonoscopy him- or herself

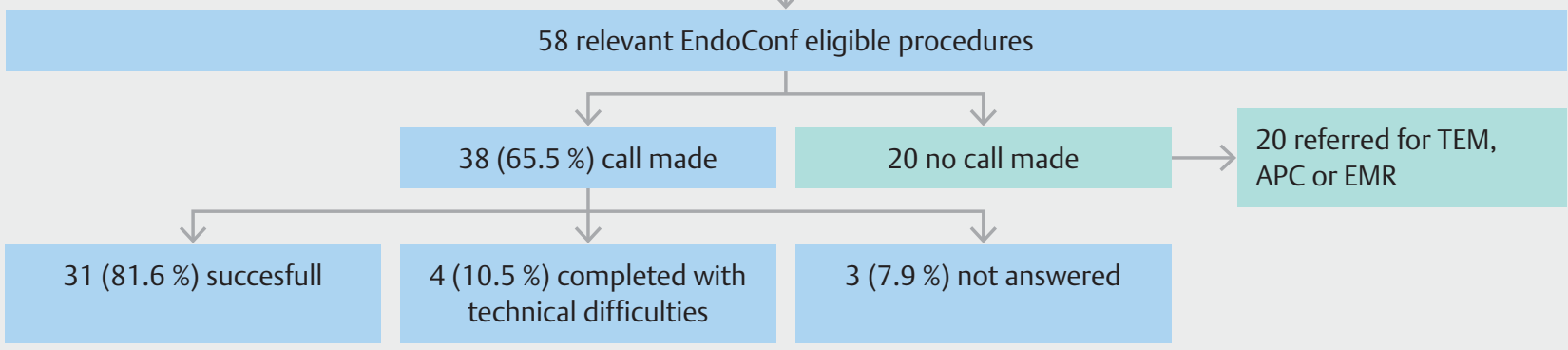

- Fig. 3 Flowchart of procedures eligible for EndoConf during 3 months of observation. TEM, transanal endoscopic microsurgery; APC, argon plasma coagulation; EMR, endoscopic mucosal resection.

further actions or doubts, a non-sedated patient could listen in, which might result in unwanted side effects such as anxiety or patient concern. Therefore, it is essential to consider how the call is conducted, although this is not any different from any physical conference or regular communication during procedures. On the other hand, patients may recognize the added value of on-the-spot extra attention and feel reassured that the decision is thought through and based on more than a single opinion. On top of this comes the possible gain of being spared readmission or maybe even complications that could occur from suboptimal procedure choices. We are also exploring the option of testing headphones for the endoscopist.

During the roll-out, several challenges need to be addressed. In $10.5 \%$ of the initiated EndoConf sessions, technical problems were experienced. However, all sessions were completed successfully. In some cases, the endoscopist or nurses were not familiar with the EndoConf equipment and setup or encountered a poor internet connection. In such cases, the endoscopist had to move the colonoscope very slowly to expose the lesion of interest for the experienced on-call endoscopist. The COVID-19 pandemic has made wearing face masks and plastic visors necessary in all settings. Several studies have reported that face masks attenuate sound and speech intelligibility, which could make EndoConf communication challenging [8,9].

Recent breakthroughs in Al have facilitated development of several systems for computer-aided detection and diagnosis, explicitly focusing on polyp detection (CADe) and polyp characterization (CADx) [10]. Potentially, Al-based systems can increase the adenoma detection rate (ADR), improve colorectal polyp diagnosis accuracy, aid in avoidance of unnecessary polypectomies, and optimize referral for correct treatment. However, the quality of the Al systems that have been developed varies markedly, and the clinical value has been questioned. A recent meta-analysis of $\mathrm{Al}$-based systems for polyp detection did not show improvement in detection of advanced polyps as compared to conventional colonoscopy [11]. The EndoConf is not expected to increase ADR in its current state. It was initially developed as a tool to aid in handling specific colorectal lesions. However, combined with Al-based systems, it may lead to further improved treatment allocation and peer education.

For a system like EndoConf to reach its full potential, it is vital to establish a few things. First, the technical quality of the call (broadband speed, audio quality, dedicated broadband, etc.) must be high; otherwise, the experience is suboptimal. Second, the acceptance of the conference type must be wide among endoscopists. It needs to be accepted by as many as possible to enhance the effect and disseminate knowledge among peers. If accepted and utilized, EndoConf holds great potential and can be implemented nationwide for experts in the field to enable RT consultations. We believe that EndoConf has the potential to be utilized internationally and in other procedures and diagnostics across surgical and medical specialties.

We are at a point where we need to implement the system in other departments, to have input from other users, and to learn what further opportunities exist for incorporating the EndoConf system to meet long-term objectives. We believe that this is a turning point in educating endoscopists about specific procedures. Use of EndoConf also is an excellent way to enhance supervision of endoscopic nurses. It is a quick and precise solution to difficult situations during which an endoscopist needs expert advice. Thus, the EndoConf system provides solutions that will prevent patients from undergoing unnecessary endoscopic procedures while ensuring that hazardous polypectomy attempts are avoided when an individual should be referred to an expert tertiary center. After 7 years of hosting the SATC teaching center, we have found that endoscopists at all 
our hospital sites are becoming more unified in their application of polypectomy techniques and how they handle advanced adenomas.

EndoConf has the potential to increase decision-making, resulting in decreased referrals for repeat endoscopies and readmissions for therapeutic procedures.

Furthermore, it has great potential for educating junior endoscopists and we suggest that this can become a standard decision-making support for inexperienced endoscopists. In the United States, an increasing rate of surgery for patients with nonmalignant $C R$ polyps has been reported and we expect that implementing EndoConf or a similar RT conference system could slow down this tendency, for the betterment of patients [4]. Our department has seen an increase in documentation of large lesions, through use of either the EndoConf system or with photo and video documenting system (Endobase, Olympus). This was a direct result of the focus placed on it during implementation of the EndoConf system.

\section{Competing interests}

The authors declare that they have no conflict of interest.

\section{References}

[1] Schreuders EH, Ruco A, Rabeneck L et al. Colorectal cancer screening: a global overview of existing programmes. Gut 2015; 64: 1637-1649
[2] Kaminski MF, Thomas-Gibson S, Bugajski M et al. Performance measures for lower gastrointestinal endoscopy: a European Society of Gastrointestinal Endoscopy (ESGE) Quality Improvement Initiative. Endoscopy 2017; 49: 378-397

[3] Ferlitsch M, Moss A, Hassan C et al. Colorectal polypectomy and endoscopic mucosal resection (EMR): European Society of Gastrointestinal Endoscopy (ESGE) Clinical Guideline. Endoscopy 2017; 49: 270-297

[4] Peery AF, Cools KS, Strassle PD et al. Increasing rates of surgery for patients with nonmalignant colorectal polyps in the United States. Gastroenterology 2018; 154: 1352-1360 e1353

[5] Rutter MD, Jover R. Personalizing Polypectomy techniques based on polyp characteristics. Clin Gastroenterol Hepatol 2020; 18: 28592867

[6] Subramaniam S, Bhandari P. Competency in polypectomy: when desirable becomes essential. Gastrointest Endosc 2018; 87: 645-647

[7] Baatrup G, Buch N. Advanced adenomas and early colorectal cancers - SATC center (SATCC). A Danish initiative for the standardization and improvement of treatment of early, significant colorectal neoplasms. Acta Oncol 2019; 58: S1-S3

[8] Porschmann C, Lubeck T, Arend JM. Impact of face masks on voice radiation. J Acoust Soc Am 2020; 148: 3663

[9] Magee M, Lewis C, Noffs G et al. Effects of face masks on acoustic analysis and speech perception: Implications for peri-pandemic protocols. J Acoust Soc Am 2020; 148: 3562

[10] Vinsard DG, Mori Y, Misawa M et al. Quality assurance of computeraided detection and diagnosis in colonoscopy. Gastrointest Endosc 2019; 90: 55-63

[11] Barua I, Vinsard DG, Jodal HC et al. Artificial intelligence for polyp detection during colonoscopy: a systematic review and meta-analysis. Endoscopy 2021; 53: 277-284 